\title{
Control of Asymmetric Cell Divisions during Root Ground Tissue Maturation
}

\author{
Ji Won Choi, and Jun Lim*
}

\begin{abstract}
Controlling the production of diverse cell/tissue types is essential for the development of multicellular organisms such as animals and plants. The Arabidopsis thaliana root, which contains distinct cells/tissues along longitudinal and radial axes, has served as an elegant model to investigate how genetic programs and environmental signals interact to produce different cell/tissue types. In the root, a series of asymmetric cell divisions (ACDs) give rise to three ground tissue layers at maturity (endodermis, middle cortex, and cortex). Because the middle cortex is formed by a periclinal (parallel to the axis) ACD of the endodermis around 7 to 14 days post-germination, middle cortex formation is used as a parameter to assess maturation of the root ground tissue. Molecular, genetic, and physiological studies have revealed that the control of the timing and extent of middle cortex formation during root maturation relies on the interaction of plant hormones and transcription factors. In particular, abscisic acid and gibberellin act synergistically to regulate the timing and extent of middle cortex formation, unlike their typical antagonism. The SHORT-ROOT, SCARECROW, SCARECROW-LIKE 3, and DELLA transcription factors, all of which belong to the plant-specific GRAS family, play key roles in the regulation of middle cortex formation. Recently, two additional transcription factors, SEUSS and GA- AND ABA-RESPONSIVE ZINC FINGER, have also been characterized during ground tissue maturation. In this review, we provide a detailed account of the regulatory networks that control the timing and extent of middle cortex formation during post-embryonic root development.
\end{abstract}

\section{INTRODUCTION}

Multicellular organisms, such as animals and plants, possess diverse cell/tissue types. How different cells and tissues are generated is one of the fundamental questions in developmen-

Department of Systems Biotechnology, Konkuk University, Seoul 05029, Korea

*Correspondence: jlim@konkuk.ac.kr

Received 21 April, 2016; revised 24 May, 2016; accepted 25 May, 2016; published online 16 June, 2016

Keywords: asymmetric cell division, ground tissue, plant hormone, root development, transcription factor tal biology. In particular, asymmetric cell divisions (ACDs) play an important role in the development of distinct cell and tissue types in the individual organism (Abrash and Bergmann, 2009; De Smet and Beeckman, 2011; Horvitz and Herskowitz, 1992; Knoblich, 2008; Smolarkiewicz and Dhonukshe, 2013; Ten Hove and Heidstra, 2008). Therefore, the timing and extent of ACDs should be controlled to ensure correct patterning. In plants, the Arabidopsis (Arabidopsis thaliana) root has been used as a model to investigate the molecular mechanisms underlying the control of ACDs in cell/tissue patterning (Benfey et al., 1993; Cruz-Ramirez et al., 2012; Cui et al., 2007; Di Laurenzio et al., 1996; Dolan et al., 1993; Helariutta et al., 2000; Scheres et al., 1994; 1995). From embryogenesis onwards, stem cells for the ground tissue (GT), namely the cortex/endodermis initial (CEI), undergo a series of ACDs. The divides in the anticlinal direction (perpendicular to the axis), resulting in self-renewal of the $\mathrm{CEI}$ and a daughter cell, i.e., the cortex/endodermis initial daughter (CEID). Next, the CEID divides in the periclinal orientation (parallel to the axis). Thus, the Arabidopsis root has two GT layers at the early stages of development: the endodermis (inside) and the cortex (outside) (Benfey et al., 1993; Cruz-Ramirez et al., 2012; Cui et al., 2007; Di Laurenzio et al., 1996; Dolan et al., 1993; Helariutta et al., 2000; Scheres et al., 1994; 1995) (Fig. 1). As the root ages, the endodermis divides again in the periclinal direction around 7 to 14 days post-germination (dpg), which results in the regeneration of the endodermis and an additional cortex. Usually, the endodermis cells adjacent to the xylem poles preferentially tend to undergo periclinal division prior to other cells in the endodermis (Baum et al., 2002; Paquette and Benfey, 2005). Due to its location between the endodermis and the pre-existing cortex in the GT layers, the new cortex layer is designated as the middle cortex (MC). Thus, the Arabidopsis root can possess three GT layers at maturity (endodermis, MC, and cortex; Fig.1B). Therefore, production of the MC layer by periclinal ACDs in the endodermis is considered to be an indication that postembryonic root development has entered a more mature phase (Baum et al., 2002; Paquette and Benfey, 2005; Pauluzzi et al., 2012). To assess maturation of the Arabidopsis root GT, the formation of the MC layer, as measured by the proportion of plants with MC either at a specific time point or in time courses, has been used (Baum et al., 2002; Cui and Benfey, 2009a; 2009b; Gong et al., 2016; Heo et al., 2011; Koizumi et al., 2012a; 2012b; Lee et al., 2016; Paquette and Benfey, 2005; Pauluzzi et al., 2012).

Since the production of the MC layer during root GT maturation was first reported (Baum et al., 2002), accumulating evi- 

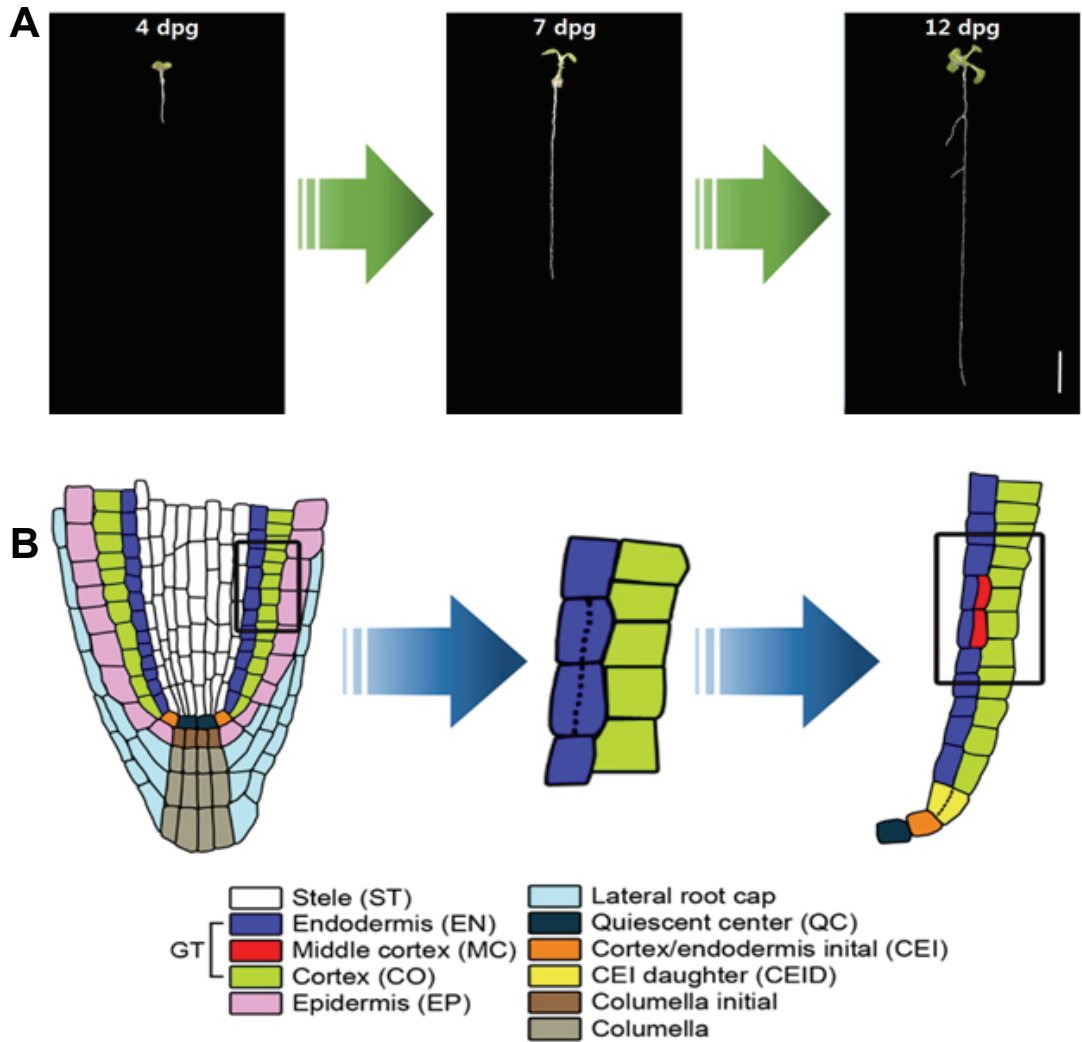

Lateral root cap Quiescent center (QC)

Cortex/endodermis inital (CEI) CEI daughter (CEID) Columella initial Columella
Fig. 1. Arabidopsis root development. (A) Arabidopsis root development under standard conditions. As the root ages, length of the primary root increases and lateral roots emerge in the later stages. Scale bar, $1 \mathrm{~cm}$. (B) Schematic presentation of the Arabidopsis root during GT maturation. The left panel illustrates a longitudinal axis of the root at an early stage in post-embryonic root development. The quiescent center (QC) and adjacent stem cells form the stem cell niche, which gives rise to cells in diverse lineages. As the root ages, the endodermis undergoes additional periclinal ACDs to generate the endodermis (EN; blue) and the middle cortex (MC; red), which is located between the endodermis and the cortex (CO; green). The right panel shows that the root has three layers in the GT: endodermis (EN), middle cortex (MC), and cortex (CO) at maturity. dence has revealed complex regulatory networks involving the interplay of plant hormones and transcription factors, which modulate the timing and extent of ACDs for MC formation. In this review, we focus on the crucial roles of plant hormones as well as transcription factors in the control of MC formation. We also describe the regulatory interactions between plant hormones and transcription factors during GT maturation. In addition, we provide perspective on other factors that potentially control the timing and extent of MC formation, and why roots develop with extra cortex layers during root GT maturation.

\section{PLANT HORMONES IN THE CONTROL OF MC FORMATION}

Under normal growth conditions, Columbia wild-type (Col WT) roots undergo periclinal ACDs in the endodermis around 7 to $14 \mathrm{dpg}$, resulting in an average of 20 to $35 \%$ of plants with the MC layer, depending on experimental conditions (Cui and Benfey, 2009a; Gong et al., 2016; Heo et al., 2011; Koizumi et al., 2012a; 2012b; Lee et al., 2016; Paquette and Benfey, 2005) Under gibberellin (GA)-deficient conditions, induced by treatment with a GA biosynthesis inhibitor (e.g., paclobutrazol; PAC) or by loss-of-function mutations in a key GA biosynthesis enzyme (e.g., ga1-3), seedling roots exhibit a 2- to 3-fold increase in MC formation (Cui and Benfey, 2009a; Gong et al., 2016; Heo et al., 2011; Koizumi et al., 2012a; 2012b; Lee et al., 2016; Paquette and Benfey, 2005). In contrast, exogenous GA application substantially suppresses periclinal ACDs in the endodermis, thus resulting in delayed MC formation (Cui and Benfey, 2009a; Gong et al., 2016; Heo et al., 2011; Koizumi et al., 2012a; 2012b; Lee et al., 2016; Paquette and Benfey, 2005). These findings indicate that modulation of bioactive GA levels is critical for the regulation of $\mathrm{MC}$ formation in the Arabidopsis root GT.

The plant hormone abscisic acid (ABA) antagonizes the effects of GA in numerous processes during plant growth and development (Finkelstein, 2013; Finkelstein et al., 2002; 2008; Hoffmann-Benning and Kende, 1992; Rohde et al., 2000; Sun and Gubler, 2004; Weiss and Ori, 2007). However, similar to seedlings treated with GA, WT roots treated with exogenous ABA exhibit a suppression of MC formation. In addition, transgenic Arabidopsis plants with XERICO fused to the $35 \mathrm{~S}$ promoter (XER overexpressor; XER-OX), which substantially increases cellular ABA levels, probably through the ubiquitin/proteasome-dependent degradation pathway (Ko et al., 2006), have almost no MC layer at $7 \mathrm{dpg}$ (Cui and Benfey, 2009a; 2009b; Lee et al., 2016). In contrast, under ABAdeficient conditions caused by loss-of-function mutations in a key ABA biosynthesis enzyme (aba2-2) or in the XER gene (xer), seedlings display more frequent periclinal ACDs for MC formation (Lee et al., 2016). Interestingly, the ABA-deficient aba2-2 and xer mutants are sensitive to PAC, resulting in the precocious formation of the MC layer. Consistent with this finding, under ABA treatment, GA-deficient ga1-3 roots show an elevated frequency of $M C$ formation when compared with WT roots. Therefore, analogous to GA, modulation of $A B A$ levels is also important for the control of root GT maturation. Taken together, these findings indicate that the bioactive levels of the two hormones play key roles in the maturation process of the root GT. However, little is known about the distribution of ABA and GA in post-embryonic root development, even though re- 
cent studies have shown that the root endodermis acts as a hub for ABA and GA responses (Dinneny, 2014; Duan et al., 2013; Heo et al., 2011; Lee et al., 2016; Miyashima and Nakajima, 2011; Shani et al., 2013; Ubeda-Tomás et al., 2008; 2009).

In contrast to what has been known to date, these findings have revealed a unique interaction between $A B A$ and $G A$, in which the two hormones act synergistically, not antagonistically, to modulate the timing and extent of MC formation (Cui and Benfey, 2009a; 2009b; Lee et al., 2016).

\section{TRANSCRIPTION FACTORS IN THE CONTROL OF MC FORMATION}

Mutations in SHORT-ROOT (SHR) and SCARECROW (SCR) were first identified over two decades ago (Benfey et al., 1993; Scheres et al., 1995). Both shr and scr mutants have fewer GT layers in the root than do WT plants. At maturity, the WT root has three layers in the GT (endodermis, MC, and cortex; Fig.1B). In shr mutants, no endodermis is found in the GT from embryogenesis onward (Benfey et al., 1993; Helariutta et al., 2000). Later in post-embryonic development, neither endodermis nor MC is formed in the shr root, which is similar to its embryos and young roots, suggesting that the endodermis layer is essential for MC formation (Cui and Benfey, 2009a; 2009b; Gong et al., 2016; Heo et al., 2011; Koizumi et al., 2012a; 2012b; Paquette and Benfey, 2005; Pauluzzi et al., 2012). In contrast, from embryogenesis to the early stages of post-embryonic development, scr mutants possess a single GT layer with mixed traits of the endodermis and cortex (Benfey et al., 1993; Cruz-Ramirez et al., 2012; Di Laurenzio et al., 1996; Heidstra et al., 2004; Scheres et al., 1994; 1995). In later postembryonic development, sporadic MC layers are precociously produced in the scr root (Cui and Benfey, 2009a; 2009b; Heo et al., 2011; Koizumi et al., 2012a; 2012b; Paquette and Benfey, 2005). Taken together, these results show that the endodermis and cortex layers in the GT fail to separate in the scr root at the early stages, whereas the scr mutant frequently undergoes periclinal ACDs for MC formation in later stages. Therefore, as the root ages, SCR has a dual role in controlling periclinal ACDs: separation of the endodermis and cortex versus MC formation. Both SHR and SCR belong to the GRAS transcription factor family, named after its original three members: GA INSENSITIVE (GAI), REPRESSOR OF GA1-3 (RGA), and SCR (Bolle, 2004; Di Laurenzio et al., 1996; Lee et al., 2008; Peng et al., 1997; Pysh et al., 1999; Silverstone et al., 1998; Tian et al., 2004). Another GRAS transcription factor, SCARECROW-LIKE 3 (SCL3), is involved in MC formation during maturation of the root GT (Heo et al., 2011; Lee et al., 2016). For example, similar to the scr mutant, scl3 shows premature $\mathrm{MC}$ formation, whereas overexpression of SCL3 suppresses periclinal ACDs in the endodermis.

Recently, two additional transcription factors have been shown to play important roles during GT maturation (Gong et al., 2016; Lee et al., 2016). SEUSS (SEU), which is known to function in reproductive development (Azhakanandam et al., 2008; Bao et al., 2010; Franks et al., 2002; Grigorova et al., 2011; Sridhar et al., 2006), is involved in the control of MC formation. In the Arabidopsis root, the seu mutant displays an increased frequency of $\mathrm{MC}$ formation, whereas overexpression of SEU results in reduced periclinal ACDs in the endodermis (Gong et al., 2016). Through transcriptomic, genetic, molecular and physiological analyses, GAZ (GA- AND ABA-RESPONSIVE ZINC FINGER), a previously uncharacterized $\mathrm{C}_{2} \mathrm{H}_{2}$-type zinc finger, has been shown to be involved in MC formation (Lee et al., 2016). Unfor- tunately, loss-of-function gaz mutants display no visible phenotype. Thus, as an alternative, transgenic Arabidopsis plants with a fusion of GAZ to the SRDX domain (GAZ-SRDX), which renders strong repressive activity of GAZ (Hiratsu et al., 2003; 2004), have been analyzed, together with RNAi lines (GAZ-RNAI) (Lee et al., 2016). In comparison with GAZ overexpression (GAZ-OX) seedlings, both GAZ-SRDX and GAZ-RNAi plants exhibit the opposite MC formation phenotypes in the hormone-mediated control of root GT maturation (described in detail below).

With only a handful of transcription factors, we currently have a glimpse of the molecular events underlying the maturation process of the root GT.

\section{REGULATORY NETWORKS INVOLVED IN THE CONTROL OF MC FORMATION}

The processes involved in MC production during Arabidopsis root maturation were first described more than a decade ago (Baum et al., 2002). Since then, the interconnected genetic and molecular mechanisms underlying the formation of the MC layer have been characterized (Cui and Benfey, 2009a; 2009b; Cui et al., 2014; Gong et al., 2016; Heo et al., 2011; Koizumi et al., 2012a; 2012b; Lee et al., 2016; Paquette and Benfey, 2005). Accumulating evidence has revealed unexpectedly complex networks of genes that play crucial roles in the regulation of $\mathrm{MC}$ formation.

In the root endodermis, SCL3 acts downstream of the SHR/SCR regulatory module during GT maturation. For example, in the scl3 shr double mutant, neither endodermis nor MC is formed, similar to the shr single mutant. In contrast, the sc/3 scr double mutant exhibits more frequent periclinal ACDs for $\mathrm{MC}$ formation than either $s c / 3$ or scr single mutants, whereas overexpression of SCL3 is sufficient to suppress the precocious $\mathrm{MC}$ formation phenotype of the scr single mutant (Heo et al., 2011). In addition to the SHR/SCR pathway, SCL3 is directly regulated by DELLA transcription factors (Heo et al., 2011; Zentella et al., 2007; Zhang et al., 2011), which are the major negative regulators of GA signaling and belong to the GRAS family (Bolle, 2004; Lee et al., 2008; Peng et al., 1997; Pysh et al., 1999; Silverstone et al., 1998; Tian et al., 2004). The gai rga double mutant shows almost no MC formation at $7 \mathrm{dpg}$, whereas the scl3 gai rga triple mutant displays an approximately 3fold increase in the frequency of periclinal ACDs for MC formation (Heo et al., 2011). Under GA-deficient conditions, the phenotype of $s c / 3$ is exacerbated, thus resulting in precocious MC formation. Taken together, SCL3 serves as a molecular link between hormonal (GA) and developmental (SHR/SCR) pathways that regulate the maturation process in post-embryonic root development (Heo et al., 2011).

Until recently, no molecular component acting upstream of SHR and SCR has been characterized in the regulation of MC formation. In seu mutants, the abundance of SHR and SCR transcripts is substantially reduced, and SEU associates with their promoter regions (Gong et al., 2016). As seen in the shr single mutant, only a single cortex is found in the seu shr double mutant. A higher frequency of $\mathrm{MC}$ formation is observed in the seu scr double mutant than in either of the single mutants. In addition to the SHR/SCR module, SEU also directly regulates transcription of SCL3 by binding to its promoter regions (Gong et al., 2016). Genetic analysis of the seu sc/3 double mutant has revealed that seu is epistatic to sc/3. Therefore, these findings indicate that SEU is involved in the formation of the MC layer by activating expression of SHR, SCR, and SCL3 during GT maturation (Gong et al., 2016). 


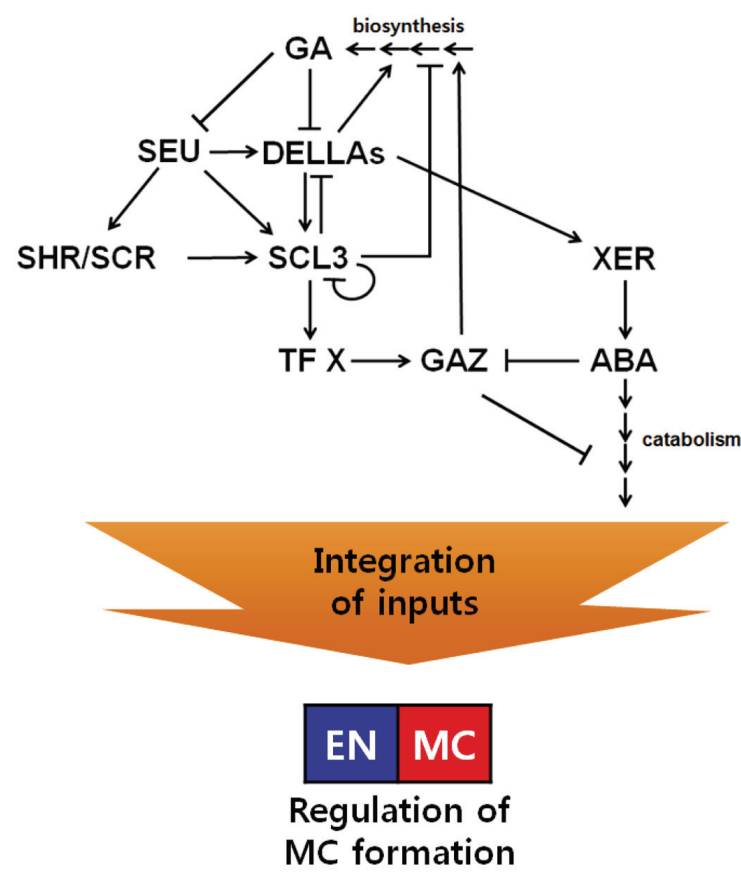

Fig. 2. Schematic model of the regulatory networks involved in MC formation. In the GA signaling pathway, bioactive GAs negatively regulate DELLA transcription factors by facilitating proteolytic degradation of DELLAs. SCL3, acting downstream of both DELLA and SHR/SCR transcription factors, serves as an endodermis-specific integrator. In addition, SEU is involved in the control of MC formation, by positively regulating the expression of $S H R, S C R$, and $S C L 3$. In parallel, the ABA pathway also controls the abundance of $G A Z$ mRNA, which plays a role in the transcriptional control of $G A$ and ABA metabolism. GAZ is positioned downstream of SCL3, which regulates the level of $G A Z$ expression via an unknown transcription factor (TF X). Thus, GAZ acts as a point of convergence for the $A B A$ and $G A$ pathways. Together, multiple inputs from plant hormone pathways (ABA and GA) and developmental pathways (SHR/SCR and SEU) should be coordinately integrated to control maturation of the Arabidopsis root GT. Arrows represent positive regulation, and bars denote negative regulation.

A recent study also has demonstrated that the GAZ transcription factor, which acts downstream of both the GA and $A B A$ pathways, plays a role in the control of $\mathrm{MC}$ formation (Lee et al., 2016). Under PAC treatment, GAZ-OX seedlings show a PACresistant phenotype, with reduced $M C$ formation. Under the same condition, GAZ-OX in the sc/3 background ( $s c / 3$ GAZ$O X$ ) has an opposite phenotype, with an increased frequency of MC formation, similar to the $s c / 3$ single mutant. Thus, the PAC-sensitive phenotype of $s c / 3 G A Z-O X$ is likely due to the loss of SCL3 function, placing GAZ downstream of SCL3 in the GA-mediated regulation of GT maturation (Lee et al., 2016). Under ABA treatment, GAZ-OX seedlings are more sensitive to ABA than are the WT seedlings, displaying almost no MC layer. Furthermore, expression of $G A Z$ is regulated by bioactive $G A$ and $A B A$ levels. For instance, the $G A Z$ transcript levels are elevated under GA deficiency induced by PAC treatment or ga1-3 mutation. In contrast, the levels of $G A Z$ expression are reduced in response to ABA treatment or by XER-OX (ABA overproducer). In addition, GAZ plays a role in the transcrip- tional regulation of $\mathrm{ABA}$ and $\mathrm{GA}$ homeostasis. Taken together, these results suggest that GAZ serves as a convergent point of the $A B A$ and $G A$ pathways during root GT maturation (Lee et al., 2016).

In summary (Fig. 2), the bioactive levels of ABA and GA play key roles in modulating the timing and extent of $M C$ formation during GT maturation. For example, high levels of $A B A$ and GA suppress the occurrence of periclinal ACDs in the endodermis, whereas mutants with ABA (aba2-2 and xer) or GA (ga1-3) deficiency have substantially increased production of the MC layer (Cui and Benfey, 2009a; 2009b; Gong et al., 2016; Heo et al., 2011; Koizumi et al., 2012a; 2012b; Lee et al., 2016; Paquette and Benfey, 2005). In the GA pathway, bioactive GAs negatively regulate DELLA proteins by promoting their degradation (Harberd et al., 2009; Jiang and Fu, 2007; Peng et al., 1997; Silverstone et al., 1998; Sun and Gubler, 2004). Downstream of DELLAs, SCL3 attenuates the activity of DELLAs by protein-protein interaction and auto-regulates its own expression (Heo et al., 2011; Zentella et al., 2007; Zhang et al., 2011). Both DELLA and SCL3 transcription factors are involved in the feedback regulation of GA biosynthesis (Heo et al., 2011; Zentella et al., 2007; Zhang et al., 2011). The DELLA proteins also promote expression of $X E R$, which is involved in the regulation of bioactive ABA levels (Ko et al., 2006; Zentella et al., 2007). Moreover, transcription of SCL3 is under the direct regulation of SHR and SCR in the endodermis (Heo et al., 2011; Levesque et al., 2006). Recently, SEU has been shown to positively regulate the expression of $S H R$ and $S C R$, and is also involved in the GA-mediated regulation of SCL3 expression (Gong et al., 2016). In addition, GAZ, through maintenance of a constant flux of $A B A$ and GA, plays a role in the control of root GT maturation. Taken together, multiple inputs from both plant hormonal (ABA and GA) and developmental (SHR, SCR, SCL3, SEU, and GAZ transcription factors) pathways should be coordinately integrated to generate the two different cell types (endodermis and $\mathrm{MC}$ ) during GT maturation (Fig. 2).

\section{CONCLUSION}

While only a single layer of endodermis exists in the GT, most plants have multiple layers of cortex in the root, with the exception of Arabidopsis (Benfey et al., 1993; Cruz-Ramirez et al., 2012; Cui et al., 2007; Di Laurenzio et al., 1996; Dolan et al., 1993; Esau, 1953; 1977; Helariutta et al., 2000; Scheres et al., 1994; 1995; Wu et al., 2014). In the plant root, the cortex layers store carbohydrates and other secondary metabolites. In addition, the root cortex is used to transport materials from the root hair into the central cylinder (Esau, 1953; 1977). Although the physiological function of MC formation is currently unclear, the presence of the multiple cortex layers generated by cell division and differentiation is thought to be a consequence of plant adaptation (Cui et al., 2014; Esau, 1953; 1977; Pauluzzi et al., 2012). For instance, rice is subject to water submergence; therefore, as an anatomical adaptation, the rice root possesses the multiple cortex layers that differentiate into gas-containing aerenchyma cells (Coudert et al., 2010; Cui et al., 2014; He et al., 1994; Rebouillat et al., 2009). In Brachypodium (Brachypodium distachyon) and rice (Oryza sativa), which contain multiple cortex layers, a plausible mechanism through which the controlled movement of the SHR transcription factor determines the number of cortex cell layers has been proposed (Wu et al., 2014). Moreover, recent work has revealed that MC formation is promoted by reactive oxygen species, suggesting that multiple cortex layers may protect against stresses (Cui, 2015; Cui 
et al., 2014). Although the number of studies investigating the regulatory networks of $\mathrm{MC}$ formation during plant root maturation has increased rapidly in recent years, our understanding of the physiological role of $\mathrm{MC}$ formation remains elusive. Future studies should aim to identify additional tissue-specific determinants and to understand how these determinants interact with known players to control MC formation. In addition, it would be of interest to investigate whether other plant hormones are involved in controlling the timing and extent of MC formation.

\section{ACKNOWLEDGMENTS}

This work was supported by grants from the National Research Foundation (NRF-2014R1A1A2057668) and the Next-Generation BioGreen21 Program (SSAC-PJ01113101). We thank members of the Lim laboratory for comments on the manuscript. We also thank Janette Lim for assisting with the artwork in the figures. We apologize to all colleagues whose work has not been mentioned due to space limitations.

\section{REFERENCES}

Abrash, E.B., and Bergmann, D.C. (2009). Asymmetric cell divisions: a view from plant development. Dev. Cell 16, 783-796.

Azhakanandam, S., Nole-Wilson, S., Bao, F., and Franks, R.G. (2008). SEUSS and AINTEGUMENTA mediate patterning and ovule initiation during gynoecium medial domain development. Plant Physiol. 146, 1165-1181.

Bao, F., Azhakanandam, S., and Franks, R.G. (2010). SEUSS and SEUSS-LIKE transcriptional adaptors regulate floral and embryonic development in Arabidopsis. Plant Physiol. 152, 821-836.

Baum, S.F., Dubrovsky, Joseph G., and Rost, Thomas L. (2002). Apical organization and maturation of the cortex and vascular cylinder in Arabidopsis thaliana (Brassicaceae) roots. Am. J. Bot. 89, 908-920.

Benfey, P.N., Linstead, P.J., Roberts, K., Schiefelbein, J.W., Hauser, M.T., and Aeschbacher, R.A. (1993). Root development in Arabidopsis: four mutants with dramatically altered root morphogenesis. Development 119, 57-70.

Bolle, C. (2004). The role of GRAS proteins in plant signal transduction and development. Planta 218, 683-692.

Coudert, Y., Perin, C., Courtois, B., Khong, N.G., and Gantet, P. (2010). Genetic control of root development in rice, the model cereal. Trends Plant Sci. 15, 219-226.

Cruz-Ramirez, A., Diaz-Trivino, S., Blilou, I., Grieneisen, V.A., Sozzani, R., Zamioudis, C., Miskolczi, P., Nieuwland, J., Benjamins, R., Dhonukshe, P., et al. (2012). A bistable circuit involving SCARECROW-RETINOBLASTOMA integrates cues to inform asymmetric stem cell division. Cell 150, 1002-1015.

Cui, H. (2015). Cortex proliferation in the root is a protective mechanism against abiotic stress. Plant Signal. Behav. 10, e1011949.

Cui, H., and Benfey, P.N. (2009a). Interplay between SCARECROW, GA and LIKE HETEROCHROMATIN PROTEIN 1 in ground tissue patterning in the Arabidopsis root. Plant J. 58, 1016-1027.

Cui, H., and Benfey, P.N. (2009b). Cortex proliferation: simple phenotype, complex regulatory mechanisms. Plant Signal. Behav. 4, 551-553.

Cui, H., Levesque, M.P., Vernoux, T., Jung, J.W., Paquette, A.J., Gallagher, K.L., Wang, J.Y., Blilou, I., Scheres, B., and Benfey, P.N. (2007). An evolutionarily conserved mechanism delimiting SHR movement defines a single layer of endodermis in plants. Science 316, 421-425.

Cui, H., Kong, D., Wei, P., Hao, Y., Torii, K.U., Lee, J.S., and Li, J. (2014). SPINDLY, ERECTA and its ligand STOMAGEN have a role in redox-mediated cortex proliferation in the Arabidopsis root. Mol. Plant 7, 1727-1739.

De Smet, I., and Beeckman, T. (2011). Asymmetric cell division in land plants and algae: the driving force for differentiation. Nat. Rev. Mol. Cell Biol. 12, 177-188.

Di Laurenzio, L., Wysocka-Diller, J., Malamy, J.E., Pysh, L., Helariutta, Y., Freshour, G., Hahn, M.G., Feldmann, K.A., and Benfey, P.N. (1996). The SCARECROW gene regulates an asymmetric cell division that is essential for generating the radial organization of the Arabidopsis root. Cell 86, 423-433.

Dinneny, J.R. (2014). A gateway with a guard: how the endodermis regulates growth through hormone signaling. Plant Sci. 214, 1419.

Dolan, L., Janmaat, K., Willemsen, V., Linstead, P., Poethig, S. Roberts, K., and Scheres, B. (1993). Cellular organization of the Arabidopsis thaliana root. Development 119, 71-84.

Duan, L., Dietrich, D., Ng, C.H., Chan, P.M., Bhalerao, R., Bennett, M.J., and Dinneny, J.R. (2013). Endodermal ABA signaling promotes lateral root quiescence during salt stress in Arabidopsis seedlings. Plant Cell 25, 324-341.

Esau, K. (1953). Plant anatomy. Wiley \& Sons, New York.

Esau, K. (1977). Anatomy of seed plants, 2nd edition. Wiley \& Sons, New York

Finkelstein, R.R. (2013). Abscisic acid biosynthesis and response. In The Arabidopsis book 11, e0166.

Finkelstein, R.R., Gampala, S.S., and Rock, C.D. (2002). Abscisic acid signaling in seeds and seedlings. Plant Cell 14 Suppl, S1545.

Finkelstein, R.R., Reeves, W., Ariizumi, T., and Steber, C. (2008). Molecular aspects of seed dormancy. Ann. Rev. Plant Biol. 59, 387-415.

Franks, R.G., Wang, C., Levin, J.Z., and Liu, Z. (2002). SEUSS, a member of a novel family of plant regulatory proteins, represses floral homeotic gene expression with LEUNIG. Development 129, 253-263.

Gong, X., Flores-Vergara, M.A., Hong, J.H., Chu, H., Lim, J., Franks, R.G., Liu, Z., and Xu, J. (2016). SEUSS integrates gibberellin signaling with transcriptional inputs from the SHR-SCR-SCL3 module to regulate middle cortex formation in the Arabidopsis root. Plant Physiol. 170, 1675-1683.

Grigorova B, Mara C, Hollender C, Sijacic P, Chen X, and Liu Z (2011) LEUNIG and SEUSS co-repressors regulate miR172 expression in Arabidopsis flowers. Development 138, 2451-2456.

Harberd, N.P., Belfield, E., and Yasumura, Y. (2009). The angiosperm gibberellin-GID1-DELLA growth regulatory mechanism: how an "inhibitor of an inhibitor" enables flexible response to fluctuating environments. Plant Cell 21, 1328-1339.

He, C.J., Drew, M.C., and Morgan, P.W. (1994). Induction of enzymes associated with Lysigenous aerenchyma formation in roots of Zea mays during hypoxia or nitrogen starvation. Plant Physiol. 105, 861-865.

Heidstra, R., Welch, D., and Scheres, B. (2004). Mosaic analyses using marked activation and deletion clones dissect Arabidopsis SCARECROW action in asymmetric cell division. Genes Dev. 18, 1964-1969.

Helariutta, Y., Fukaki, H., Wysocka-Diller, J., Nakajima, K., Jung, J., Sena, G., Hauser, M.T., and Benfey, P.N. (2000). The SHORTROOT gene controls radial patterning of the Arabidopsis root through radial signaling. Cell 101, 555-567.

Heo, J.-O., Chang, K.S., Kim, I.A., Lee, M.-H., Lee, S.A., Song, S.K., Lee, M.M., and Lim, J. (2011). Funneling of gibberellin signaling by the GRAS transcription regulator SCARECROW-LIKE 3 in the Arabidopsis root. Proc. Natl. Acad. Sci. USA 108, 2166-2171.

Hiratsu, K., Matsui, K., Koyama, T., and Ohme-Takagi, M. (2003). Dominant repression of target genes by chimeric repressors that include the EAR motif, a repression domain, in Arabidopsis. Plant J. 34, 733-739.

Hiratsu, K., Mitsuda, N., Matsui, K., and Ohme-Takagi, M. (2004) Identification of the minimal repression domain of SUPERMAN shows that the DLELRL hexapeptide is both necessary and sufficient for repression of transcription in Arabidopsis. Biochem. Biophys. Res. Commun. 321, 172-178.

Hoffmann-Benning, S., and Kende, H. (1992). On the role of abscisic acid and gibberellin in the regulation of growth in rice. Plant Physiol. 99, 1156-1161.

Horvitz, H.R., and Herskowitz, I. (1992). Mechanisms of asymmetric cell division: two Bs or not two Bs, that is the question. Cell 68 237-255.

Jiang, C., and Fu, X. (2007). GA action: turning on de-DELLA repressing signaling. Curr. Opin. Plant Biol. 10, 461-465.

Knoblich, J.A. (2008). Mechanisms of asymmetric stem cell division. Cell 132, 583-597.

Ko, J.H., Yang, S.H., and Han, K.H. (2006). Upregulation of an Arabidopsis RING-H2 gene, XERICO, confers drought tolerance through increased abscisic acid biosynthesis. Plant J. 47, 343- 
355.

Koizumi, K., Hayashi, T., and Gallagher, K.L. (2012a). SCARECROW reinforces SHORT-ROOT signaling and inhibits periclinal cell divisions in the ground tissue by maintaining SHR at high levels in the endodermis. Plant Signal. Behav. 7, 1573-1577.

Koizumi, K., Hayashi, T., Wu, S., and Gallagher, K.L. (2012b). The SHORT-ROOT protein acts as a mobile, dose-dependent signal in patterning the ground tissue. Proc. Natl. Acad. Sci. USA 109, 13010-13015.

Lee, M.-H., Kim, B., Song, S.K., Heo, J.O., Yu, N.I., Lee, S.A., Kim, M., Kim, D.G., Sohn, S.O., Lim, C.E., et al. (2008). Large-scale analysis of the GRAS gene family in Arabidopsis thaliana. Plant Mol. Biol. 67, 659-670.

Lee, S.A., Jang, S., Yoon, E.K., Heo, J.-O., Chang, K.S., Choi, J.W., Dhar, S., Kim, G., Choe, J.E., Heo, J.B., et al. (2016). Interplay between $\mathrm{ABA}$ and $\mathrm{GA}$ modulates the timing of asymmetric cell divisions in the Arabidopsis root ground tissue. Mol. Plant 9, 870884.

Levesque, M.P., Vernoux, T., Busch, W., Cui, H., Wang, J.Y., Blilou, I., Hassan, H., Nakajima, K., Matsumoto, N., Lohmann, J.U., et al. (2006). Whole-genome analysis of the SHORT-ROOT developmental pathway in Arabidopsis. PLoS Biol. 4, e143.

Miyashima, S., and Nakajima, K. (2011). The root endodermis: a hub of developmental signals and nutrient flow. Plant Signal. Behav. 6, 1954-1958.

Paquette, A.J., and Benfey, P.N. (2005). Maturation of the ground tissue of the root is regulated by gibberellin and SCARECROW and requires SHORT-ROOT. Plant Physiol. 138, 636-640.

Pauluzzi, G., Divol, F., Puig, J., Guiderdoni, E., Dievart, A., and Périn, C. (2012). Surfing along the root ground tissue gene network. Dev. Biol. 365, 14-22.

Peng, J., Carol, P., Richards, D., King, K., Cowling, R., Murphy, G., and Harberd, N. (1997). The Arabidopsis GAl gene defines a signaling pathway that negatively regulates gibberellin responses. Genes Dev. 11, 3194-3205.

Pysh, L.D., Wysocka-Diller, J.W., Camilleri, C., Bouchez, D., and Benfey, P.N. (1999). The GRAS gene family in Arabidopsis: sequence characterization and basic expression analysis of the SCARECROW-LIKE genes. Plant J. 18, 111-119.

Rebouillat, J., Dievart, A., Verdeil, J., Escoute, J., Giese, G., Breitler, J., Gantet, P., Espeout, S., Guiderdoni, E., and Périn, C. (2009). Molecular genetics of rice root development. Rice 2, 15-34.

Rohde, A., Kurup, S., and Holdsworth, M. (2000). ABI3 emerges from the seed. Trends Plant Sci. 5, 418-419.

Scheres, B., Wolkenfelt, H., Willemsen, V., Terlouw, M., Lawson, E., Dean, C., and Weisbeek, P. (1994). Embryonic origin of the Arabidopsis primary root and root meristem initials. Development 120, 2475-2487.

Scheres, B., Di Laurenzio, L., Willemsen, V., Hauser, M.T., Janmaat, K., Weisbeek, P., and Benfey, P.N. (1995). Mutations affecting the radial organization of the Arabidopsis root display specific defects throughout the embryonic axis. Development 121, 53-62.

Shani, E., Weinstain, R., Zhang, Y., Castillejo, C., Kaiserli, E., Chory J., Tsien, R.Y., and Estelle, M. (2013). Gibberellins accumulate in the elongating endodermal cells of Arabidopsis root. Proc. Natl. Acad. Sci. USA 110, 4834-4839.

Silverstone, A., Ciampaglio, C., and Sun, T. (1998). The Arabidopsis RGA gene encodes a transcriptional regulator repressing the gibberellin signal transduction pathway. Plant Cell 10, 155-169.

Smolarkiewicz, M., and Dhonukshe, P. (2013). Formative cell divisions: principal determinants of plant morphogenesis. Plant Cell Physiol. 54, 333-342.

Sridhar VV, Surendrarao A, and Liu Z (2006) APETALA1 and SEPALLATA3 interact with SEUSS to mediate transcription repression during flower development. Development 133, 31593166.

Sun, T.P., and Gubler, F. (2004). Molecular mechanism of gibberellin signaling in plants. Ann. Rev. Plant Biol. 55, 197-223.

Ten Hove, C.A., and Heidstra, R. (2008). Who begets whom? Plant cell fate determination by asymmetric cell division. Curr. Opin. Plant Biol. 11, 34-41.

Tian, C., Wan, P., Sun, S., Li, J., and Chen, M. (2004). Genomewide analysis of the GRAS gene family in rice and Arabidopsis. Plant Mol. Biol. 54, 519-532.

Ubeda-Tomás, S., Swarup, R., Coates, J., Swarup, K., Laplaze, L., Beemster, G.T., Hedden, P., Bhalerao, R., and Bennett, M.J. (2008). Root growth in Arabidopsis requires gibberellin/DELLA signaling in the endodermis. Nat. Cell Biol. 10, 625-628.

Ubeda-Tomás, S., Federici, F., Casimiro, I., Beemster, G.T., Bhalerao, R., Swarup, R., Doerner, P., Haseloff, J., and Bennett, M.J. (2009). Gibberellin signaling in the endodermis controls Arabidopsis root meristem size. Curr. Biol. 19, 1194-1199.

Weiss, D., and Ori, N. (2007). Mechanisms of cross talk between gibberellin and other hormones. Plant Physiol. 144, 1240-1246.

Wu, S., Lee, C.M., Hayashi, T., Price, S., Divol, F., Henry, S., Pauluzzi, G., Perin, C., and Gallagher, K.L. (2014). A plausible mechanism, based upon SHORT-ROOT movement, for regulating the number of cortex cell layers in roots. Proc. Natl. Acad. Sci. USA 111, 16184-16189.

Zentella, R., Zhang, Z.L., Park, M., Thomas, S.G., Endo, A., Murase, K., Fleet, C.M., Jikumaru, Y., Nambara, E., Kamiya, Y., et al. (2007). Global analysis of DELLA direct targets in early gibberellin signaling in Arabidopsis. Plant Cell 19, 3037-3057.

Zhang, Z.L., Ogawa, M., Fleet, C.M., Zentella, R., Hu, J., Heo, J.-O., Lim, J., Kamiya, Y., Yamaguchi, S., and Sun, T.P. (2011). SCARECROW-LIKE 3 promotes gibberellin signaling by antagonizing master growth repressor DELLA in Arabidopsis. Proc. Natl. Acad. Sci. USA 108, 2160-2165. 